\title{
INFLUENCIA DE LAS EXPECTATIVAS SOBRE LA FUERZA DE PRENSIÓN MANUAL EN SUJETOS ASINTOMÁTICOS. ENSAYO PILOTO ALEATORIZADO A SIMPLE CIEGO
}

\author{
Ferran Cuenca-Martínez, MSc ${ }^{1,2}$; Luis Suso-Martí, MSc ${ }^{1,2}$; Cristina Cabañas-García, PT ${ }^{1}$; \\ Alfonso Gil-Martínez, $\mathrm{PhD}^{1,3}$. \\ 1. Departamento de Fisioterapia, Centro Superior de Estudios Universitarios La Salle, Universidad Autónoma de Madrid, España. \\ 2. Grupo de Investigación Motion in Brains, Instituto de Neurociencia y Ciencias del Movimiento (INCIMOV), \\ Centro Superior de Estudios Universitarios La Salle, Universidad Autónoma de Madrid, España. \\ 3. Instituto de Investigación Sanitaria del Hospital Universitario La Paz (IdiPAZ), Madrid, España
}

\section{Correspondencia:}

Alfonso Gil-Martínez, PT, PhD. Facultad de Ciencias de la Salud. CESU La Salle. Universidad Autónoma of Madrid. Calle La Salle, nº 10, 28023 Madrid, España Teléfono: (+34) 917401980

Fax: (+34) 913571730 E-Mail: alfonso.gil@idipaz.es

\section{Conflicto de Intereses:}

Los autores del presente manuscrito declaran no presentar ningún conflicto de interés y además el presente estudio no ha sido presentado en ningún evento científico.

Financiación

Los autores declaran no haber recibido financiación/compensación para el desarrollo de esta investigación.

Comité de Ética:

CSEULS -PI-007/2013

DOI:

https://doi.org/10.37382/jomts.v1i1.9

Recepción del Manuscrito:

20-Diciembre-2018

Aceptación del Manuscrito:

2- Abril-2019

Licensed under:

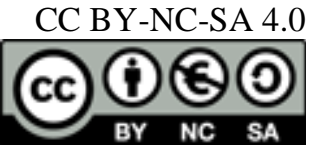

\section{RESUMEN}

Objetivo: Evaluar cómo las expectativas, inducidas de forma escrita, influyen en la fuerza de prensión manual, en la intensidad de dolor y en variables somato-sensoriales.

Diseño: Ensayo piloto controlado y aleatorizado a simple ciego

Marco: Campus Universitario.

Participantes: Los criterios de inclusión fueron: sujetos sanos, sin dolor y en edades comprendidas entre 18 y 30 años. Una muestra total de 39 sujetos sanos participaron en el presente estudio piloto y fueron asignados aleatoriamente en tres grupos según la expectativa, la cual podía ser positiva, negativa o neutra, dada por escrito

Intervenciones: Tras asignar la muestra de manera aleatoria a los tres grupos de expectativas, se les aplicó una corriente de neuro-estimulación eléctrica transcutánea (TENS) de corta duración y sin efecto terapéutico.

Variables: La variable principal fue la fuerza de prensión manual. Las variables secundarias fueron la intensidad de dolor, los umbrales de dolor a la presión y la sumación temporal en zona epicondilar. En primer lugar, se recogieron los valores pre-intervención y tras la intervención, se recogieron los datos de las variables de interés en dos tiempos. Primero, inmediatamente después de la intervención y segundo, a los 5 minutos de la misma.

Resultados: No se encontraron diferencias estadísticamente significativas en relación a la influencia de las expectativas con el cambio de la fuerza de prensión manual ( $p>0,05$ ). No se encontraron diferencias estadísticamente significativas en la sumación temporal, ni en los umbrales de dolor a la presión. El dolor post-intervención fue mayor en el grupo de expectativas negativas y este fue el único grupo que mantuvo el dolor a los 5 minutos tras finalizar la intervención.

Conclusión: A pesar de las tendencias observadas, no se encontraron influencias estadísticamente significativas de las expectativas sobre la fuerza muscular de prensión manual, ni en la intensidad de dolor, ni en variables somato-sensoriales. Las expectativas negativas influyeron en el dolor post-intervención.

Palabras Clave: Expectativas, Fuerza de prensión manual, TENS sin acción terapéutica, intensidad de dolor. 


\section{INTRODUCCIÓN}

Influencias negativas tanto en la esfera cognitivoevaluadora (Campbell et al., 2010; Verhagen et al., 2010), como en la motivacional-afectiva (Tsai et al., 2012) pueden provocar, en ámbito clínico, tanto un aumento de la experiencia dolorosa, como una disminución del éxito de los posibles tratamientos enfocados a la disminución de la percepción de la misma (Saavedra-Hernández et al., 2012).

Es por lo tanto, que la respuesta de un paciente ante un tratamiento va a estar influenciada por las expectativas que el paciente pueda tener o recibir antes de iniciar el mismo (Younger et al., 2012; Faasse \& Petrie, 2013). Se ha reportado que ante distintas modalidades de tratamiento, únicamente los pacientes que presentaron expectativas positivas ante este, mostraron mayores niveles de funcionalidad, independientemente del tratamiento al que fueron sometidos (Kalauokalani et al., 2001).

En relación a esto, experimentalmente se ha observado que el efecto de una expectativa positiva, ante un estímulo de dolor intenso, fue provocar una disminución de la percepción dolorosa y contrariamente, la inducción de una expectativa negativa ante un estímulo mínimamente doloroso fue capaz de provocar un aumento de la percepción del mismo (Ružić et al., 2017).

Por otro lado, las variables psicosociales no solamente se encuentran relacionadas en los procesos implicados en la modulación del dolor y en la respuesta a los tratamientos, sino también, se ha reportado en la literatura científica que pueden tener una influencia sobre variables físicas tales como la percepción de la fatiga, la generación de la fuerza o la adquisición de nuevas habilidades motoras (Jensen et al., 2005; Noakes et al., 2005). De hecho, en presencia de síntomas depresivos o pensamientos catastróficos, se han obtenido una disminución, tanto en la fuerza isométrica en pruebas funcionales de extensión lumbar, como en la fuerza isocinética de prensión palmar (Papciak \& Feuerstein, 1991; Watson \& Ring, 2008).
Es por lo tanto que bajo un paradigma biopsicosocial, influencias tanto a nivel cognitivo, como a nivel afectivo-emocional y en consecuencia a nivel conductual, como pudiera ser una expectativa ante un tratamiento, podría tener una influencia a considerar, no solamente en la modulación del dolor y en la disminución de la discapacidad, sino también en relación a variables funcionales y físicas.

Sin embargo, no se ha estudiado en profundidad el potencial de las expectativas en población no clínica así como su posible influencia en variables de tipo físicas, funcionales y somato-sensoriales. Es por lo tanto que el objetivo principal del presente estudio fue evaluar la influencia de expectativas, inducidas de forma escrita, sobre la fuerza de prensión manual en sujetos sanos tas la aplicación de corriente de neuroestimulación eléctrica transcutánea (TENS) sin efecto terapéutico. Un efecto secundario muy común tras la aplicación de este tipo de corriente es la aparición de dolor post-intervención (Palmer et al., 1999). Es por lo tanto que el objetivo secundario fue valorar si existe alguna influencia de las expectativas dadas sobre la intensidad dolorosa reportada postintervención así como sobre variables somatosensoriales.

\section{MÉTODOS}

\section{Diseño de estudio}

Este estudio fue un ensayo piloto controlado, aleatorizado a simple ciego. El estudio se planificó y realizó de acuerdo con los requisitos de la declaración CONSORT (normas consolidadas para diseñar y publicar ensayos clínicos aleatorizados)

\section{Participantes}

Una muestra de sujetos sanos fue obtenida del Centro Superior de Estudios Universitarios de La Salle y de la Comunidad de Madrid a través de anuncios en distintas redes sociales, así como en pósteres y correos electrónicos. Los participantes fueron reclutados entre Febrero y Julio de 2016. 
Los criterios de inclusión fueron: sujetos sanos, sin dolor y en edades comprendidas entre 18 y 30 años. Los criterios de exclusión fueron: a) sujetos con dolor en el momento del estudio, b) menores de edad, c) presencia de enfermedades sistémicas, neurológicas, cardio-respiratorias o musculo-esqueléticas, y d) sujetos sometidos a intervenciones quirúrgicas en los miembros superiores.

Se obtuvo el consentimiento informado y por escrito de todos los sujetos antes de la inclusión. Todos los participantes recibieron una explicación sobre los procedimientos del estudio los cuales, fueron planificados bajo las normas éticas de la Declaración de Helsinki y fueron aprobados por el comité de ética del Centro Superior de Estudios Universitarios de La Salle.

\section{Aleatorización}

La aleatorización se realizó utilizando una tabla de secuencias aleatorias generada por ordenador con un diseño de tres bloques equilibrados (GraphPad Software, Inc., CA, EE.UU.). Un estadístico generó la lista de asignación al azar y una vez que la evaluación inicial y la inclusión de los participantes fueron completadas, los participantes incluidos fueron asignados aleatoriamente a cualquiera de los tres grupos usando la lista de secuencia aleatoria.

\section{Enmascaramiento}

Un evaluador independiente y cegado llevó a cabo las mediciones y la recogida de los datos sin conocer el grupo al que pertenecía cada participante. Adicionalmente, los participantes fueron instruidos para que no realizasen ningún comentario al investigador que recogía las mediciones.

\section{Variable Principal}

\section{- Fuerza de prensión palmar}

Para valorar la fuerza de prensión palmar se utilizó el dinamómetro isocinético Jamar. El dinamómetro isocinético es un instrumento destinado al control estandarizado de características del rendimiento muscular tales como la producción de momento de fuerza, amplitud del movimiento, velocidad de desarrollo de la tensión y los intervalos de tiempo entre las acciones recíprocas del músculo (Stark et al., 2011). El dinamómetro Jamar presenta excelente fiabilidad intra-evaluador $(\mathrm{CCI}=0,94$ y 0,98$)$ y excelente fiabilidad inter-examinador $(\mathrm{CCI}=0,98)$ (Peolsson et al., 2001).

\section{Variables Secundarias}

- Intensidad de dolor

El objetivo de registrar la variable intensidad de dolor reside en que la corriente TENS convencional, con frecuencia entre 50 y $150 \mathrm{~Hz}$ y con duración de impulso entre 0,04 y 0,02 milisegundos, es una corriente que puede provocar, como efecto adverso, una irritación tópica local en la zona donde se sitúan los electrodos y en consecuencia puede dar lugar a molestia o dolor (Deyo et al., 1990; Palmer et al., 1999).

Es por lo tanto que, ante la posibilidad de que aparezca dicho efecto adverso, se cuantificó la intensidad de dolor post-intervención. La intensidad del dolor se valoró utilizando la escala visual analógica (EVA). Fue diseñada para valorar el dolor de una forma subjetiva. Esta escala consiste en una línea recta de 100 milímetros de longitud en cuyos extremos se representan los límites de la intensidad del dolor ("sin dolor" a "máximo dolor experimentado alguna vez"). La escala EVA ha mostrado tener buena fiabilidad T-retest $(r=0,94)$ (Bijur et al., 2001).

\section{- Umbral de dolor a la presión}

Un algómetro digital, (modelo Fx. 25 Force Gage, Wagner Instruments, Greenwich, CT, USA), se utilizó para medir el umbral de dolor a la presión (UDP), el cual, es un dinamómetro validado que permite medir la presión que se ejerce sobre una superficie de un centímetro, en una escala de 0,2 a $10 \mathrm{~kg} / \mathrm{cm} 2$ (Fischer, 1987; Chesterton et al., 2007).

\section{- Sumación temporal}

La sumación temporal se midió a partir de los monofilamentos Von Frey. Los filamentos de Von Frey consisten en un conjunto de filamentos de diferente dureza y textura que con presión graduada sirven para evaluar la sensibilidad mecánica al estímulo táctil (Starkweather et al., 2016). 


\section{- Niveles de catastrofismo ante el dolor}

Los niveles de catastrofismo ante el dolor se midieron a partir de la escala de catastrofismo ante el dolor (ECD), con la versión validada al español. La ECD es una escala autoadministrada de 13 ítems y una de las más utilizadas para valorar el constructo "catastrofización ante el dolor". Comprende 3 dimensiones: a) rumiación, b) magnificación, y c) desesperanza.

Es una escala Líkert de 5 puntos que va de 0 (nunca) a 4 (siempre). De la escala se obtiene una puntuación total que refleja el nivel de catastrofismo ante el dolor del sujeto. El intervalo teórico del instrumento se sitúa entre 0 y 52, indicando las puntuaciones bajas escaso catastrofismo, y los valores altos, elevado catastrofismo (García-Campayo et al., 2008).

\section{- Miedo al dolor}

Para cuantificar el miedo al dolor se utilizó el cuestionario del miedo al dolor (FPQ). El FPQ es un cuestionario que evalúa los miedos a una variada naturaleza de estímulos y situaciones que pueden producir dolor. Consta de 30 ítems que aportan una estructura de 3 factores: grandes miedos, miedos de escasa intensidad y los dentales. Ha mostrado poseer buenas propiedades psicométricas (Solé et al., 2017).

\section{- Niveles de ansiedad}

Los niveles de ansiedad se midieron a través del cuestionario del estado de ansiedad adaptado y validado al español (STAI). Las puntuaciones del STAI varían desde un mínimo de 0 hasta un máximo de 60 puntos. Se evalúa en una escala que va de 0 a 3 puntos en cada ítem. Una vez obtenidas las puntuaciones directas, se trasladan a la Tabla de Baremos, de la que se obtiene los niveles de ansiedad (Fonseca-Pedrero et al., 2012).

\section{Procedimiento}

La intervención sobre cada participante tuvo una duración aproximada de 45 minutos. Los participantes entraron en una sala tranquila, luminosa, y con una temperatura agradable. En primer lugar, a cada participante, se le informó de los procedimientos del estudio y firmó por escrito el consentimiento informado. Posteriormente se recogieron los datos socio-demográficos y se pasó una batería de cuestionarios para registrar la información de la esfera psicosocial con el objetivo de verificar que todos los participantes tuviesen una similar condición psicológica.

A continuación, se realizaron las mediciones de la fuerza de prensión palmar en la mano dominante con el dinamómetro, donde los participantes se colocaron en la posición de sedestación con el antebrazo apoyado en la camilla. La indicación fue que se realizasen los gestos "con la máxima fuerza posible". Posteriormente se midió el UDP, $4 \mathrm{~cm}$ distal al epicóndilo lateral del brazo dominante. Los participantes se colocaron de forma cómoda en sedestación, y se marcó con un círculo el punto de medición para usarlo como marcador en posteriores mediciones. Fue notificado que se iba a aplicar una presión con el algómetro donde en un principio se notaría una sensación de presión y que en un momento determinado se transformaría en percepción dolorosa. En ese instante se debería comunicar al examinador para que detuviese la presión ejercida y dicho valor fue tomado, haciendo hincapié en que no se debería de aguantar el dolor.

Por último, se midió la sumación temporal de estímulos en la eminencia tenar de la mano dominante con los monofilamentos de Von Frey, graduados de 0,03 a 75 g. La medición se realizó dentro de $1 \mathrm{~cm} 2$ de piel en la eminencia tenar. Se realizó una presión y se les pasó a los participantes la escala EVA, y posteriormente con la ayuda de un metrónomo se realizó una sumación temporal de 10 estímulos sobre el mismo punto a un ritmo de 60 pulsaciones por minuto; inmediatamente después se volvió a pasar la escala EVA. Esto se realizó en tres ocasiones. Finalmente, el valor final se calculó como la diferencia entre la calificación media de las tres repeticiones de un estímulo y la calificación media de las tres repeticiones de 10 estímulos.

Tras ello, se dio a los participantes un sobre que contenía una de las tres expectativas incluidas en el 
estudio. Estas podían ser positivas, negativas o neutras. A los participantes en el grupo de las expectativas positivas, el sobre contenía una nota con la siguiente información: "Se le va a aplicar una técnica de electroterapia de alta frecuencia que puede aumentar su fuerza muscular".

A los participantes del grupo de expectativas neutras, el sobre contenía un escrito que decía: "Se le va a aplicar una técnica de electroterapia de alta frecuencia que hasta ahora no se sabe cómo va a actuar sobre la fuerza muscular".

Finalmente, a los participantes del grupo de expectativas negativas se les dio otro sobre, cuya nota tenía escrito: "Se le va a aplicar una técnica de electroterapia de alta frecuencia que puede disminuir su fuerza muscular".

Tras esto, a los participantes incluidos en el estudio se les aplicó una corriente TENS durante 5 minutos con una frecuencia de $60 \mathrm{~Hz}$, a una intensidad variable, lo suficiente para que los participantes la percibiesen, pero sin llegar a provocar contracción muscular, con el electrodo negativo en el dorso de la muñeca, $2 \mathrm{~cm}$ debajo de la estiloides cubital; y el electrodo positivo en la arcada de Frohse, $2 \mathrm{~cm}$ por debajo del epicóndilo lateral del brazo dominante.

Tras ello se volvieron a tomar las mediciones de manera idéntica a las tomadas en el momento preintervención. Se realizaron dos mediciones postintervención, una inmediatamente después de finalizar la intervención y una segunda medición a los 5 minutos de finalizar la misma.

\section{Análisis estadístico}

El análisis estadístico fue llevado a cabo a través del software SPSS v. 22.0 (SPSS Inc., Chicago, IL, USA). Para el análisis de los datos, se utilizó un intervalo de confianza del 95\%, considerándose el valor $\mathrm{p}<0,05$ como estadísticamente significativo. Se utilizó el test de Shapiro-Wilk para comprobar la distribución normal de los datos. Se usó el test de ANOVA para comparar las medias de las variables demográficas y las variables de control entre los tres grupos. También se utilizó el test de ANOVA sobre las variables de interés para observar las comparaciones inter e intra-grupo tanto en el momento pre, como el momento post, así como a los 5 minutos tras finalizar la intervención.

El tamaño del efecto (d de Cohen) fue calculado para la variable principal. De acuerdo con el método Cohen, el efecto fue considerado pequeño $(0,20$ a $0,49)$, medio $(0,50$ a 0,79$)$ o grande $(>0,80)$ (Cohen, 1988).

\section{RESULTADOS}

Un total de 39 participantes fueron incluidos en el presente estudio y fueron asignados aleatoriamente a tres grupos equilibrados de 13 participantes por cada grupo. No hubo abandonos reportados en ninguno de los tres grupos, sin embargo, se encontraron eventos adversos en el mismo. Un 28,2\% de los participantes refirieron dolor tras la aplicación de la corriente TENS. El grupo sometido a expectativas positivas mostró una puntuación de $13 \pm 1,4 \mathrm{~mm}$ en la escala de EVA, en el grupo de expectativas neutras fue de $21 \pm$ 1,5 y finalmente el grupo de expectativas negativas mostraron una puntuación de $28 \pm 2,5$. Estas diferencias inter-grupos no fueron estadísticamente significativas $(p>0,05)$. Un 7,7\% de los participantes mantuvieron el dolor post-intervención hasta los 5 minutos de la intervención, aunque solamente en el grupo sujeto a expectativas negativas $(12 \pm 1,7)$.

No se encontraron diferencias estadísticamente significativas en los datos socio-demográficos y en las medidas de auto-registro (Tabla 1). Todas las variables se distribuyeron de manera normal ( $\mathrm{p}>$ $0,05)$ (Tabla 2).

En relación a la variable principal, se encontraron cambios en la fuerza muscular del antebrazo y mano tras la intervención en el grupo sometido a expectativas positivas. Por el contrario se encontró un descenso de la fuerza muscular en el grupo sujeto a expectativas negativas aunque estos cambios no fueron estadísticamente significativos en ninguno de los dos grupos $(\mathrm{p}>0,05)$. 
Tabla 1. Estadísticos descriptivos de las variables sociodemográficas.

\begin{tabular}{|c|c|c|c|c|}
\hline Variables & GPs & GNt & GNg & P-valor \\
\hline Edad (años) & $\begin{array}{c}25,31 \pm \\
2,81\end{array}$ & $\begin{array}{c}26,08 \pm \\
3,98\end{array}$ & $\begin{array}{c}26,00 \pm \\
3,00\end{array}$ & 0,809 \\
\hline Altura (m) & $\begin{array}{c}1,71 \pm \\
0,08\end{array}$ & $\begin{array}{c}1,70 \pm \\
0,07\end{array}$ & $\begin{array}{c}1,71 \pm \\
0,09\end{array}$ & 0,903 \\
\hline Peso (Kg) & $\begin{array}{c}65,69 \pm \\
9,27\end{array}$ & $\begin{array}{c}62,04 \pm \\
13,22\end{array}$ & $\begin{array}{c}62,77 \pm \\
11,24\end{array}$ & 0,689 \\
\hline Género & & & & 0,682 \\
\hline Masculino & $7(53,8)$ & $5(38,5)$ & $7(53,8)$ & \\
\hline Femenino & $6(46,2)$ & $8(61,5)$ & $6(46,2)$ & \\
\hline Nivel Educativo & & & & 0,150 \\
\hline Primario & $0(0,0)$ & $0(0,0)$ & $0(0,0)$ & \\
\hline Secundario & $2(15,4)$ & $4(40,0)$ & $1(60,0)$ & \\
\hline Universitario & $11(84,6)$ & $9(60,0)$ & $12(40,0)$ & \\
\hline $\begin{array}{l}\text { Los datos son pr } \\
\text { El nivel de signi } \\
\text { Abreviaturas: } \\
\text { expectativas neu }\end{array}$ & $\begin{array}{l}\text { ados como } \\
\text { ón fue } 0,0 \\
\text { rupo de e } \\
\text { GNg: grup }\end{array}$ & lia \pm des & $\begin{array}{l}\text { as; GNt: } \\
\text { negativa }\end{array}$ & dero $(\%)$. \\
\hline
\end{tabular}

No se halló ningún cambio en el grupo sometido a la expectativa neutra (Tabla 3 ).

No se encontraron diferencias estadísticamente significativas en la sumación temporal entre los tres grupos de expectativa ni en los UDP ( $p>0,05)$.
Los sujetos de los tres grupos de intervención presentaron similares características psicosociales, obteniéndose bajos niveles de catastrofismo, de miedo al dolor y del estado de ansiedad.

\section{DISCUSIÓN}

El objetivo principal del presente estudio fue comprobar el papel de las expectativas positivas, neutras o negativas tras la aplicación de una corriente TENS $\sin$ efecto terapéutico sobre la fuerza de prensión manual. El objetivo secundario fue analizar esta influencia sobre la intensidad de dolor, el UDP y la sumación temporal. Los resultados del presente estudio sugieren que las expectativas pueden tener una influencia sobre la fuerza muscular, sin embargo, estas diferencias no fueron estadísticamente significativas intra e inter-grupo. De la misma forma, no se obtuvieron diferencias estadísticamente significativas en la intensidad de dolor, en el UDP, ni en la sumación temporal.

\section{Fuerza de prensión manual}

Existe una carencia de literatura científica en relación al papel que pueden desempeñar las expectativas en la modificación de variables físicas tales como la fuerza muscular, la fatiga o el rango de movimiento. Sin embargo, Kalasountas et al. (2007) observaron cómo las expectativas positivas respecto a la toma de un placebo oral ocasionaron un aumento

Tabla 2. Valores pre-intervención de las medidas de auto-informe y las variables físicas.

\begin{tabular}{|c|c|c|c|c|c|c|}
\hline & Fuerza (Kg) & UDP $\left(\mathrm{kg} / \mathrm{cm}^{2}\right)$ & ST & ECD & FPQ & STAI \\
\hline GPs & $35,7 \pm 8,42$ & $1,64 \pm 0,44$ & $1,38 \pm 0,74$ & $16,31 \pm 8,5$ & $73,77 \pm 17,5$ & $11,92 \pm 5,7$ \\
\hline GNt & $30,7 \pm 11,6$ & $1,65 \pm 0,53$ & $1,61 \pm 1,16$ & $15,7 \pm 7,04$ & $79,23 \pm 14,15$ & $9,38 \pm 5,82$ \\
\hline GNg & $33,3 \pm 11,4$ & $1,27 \pm 0,41$ & $1,38 \pm 0,84$ & $16,7 \pm 5,4$ & $74,9 \pm 9,5$ & $13,15 \pm 13,8$ \\
\hline P-valor & 0,487 & 0,077 & 0,768 & 0,936 & 0,586 & 0,57 \\
\hline
\end{tabular}


Tabla 3. Análisis comparativo de la variable principal.

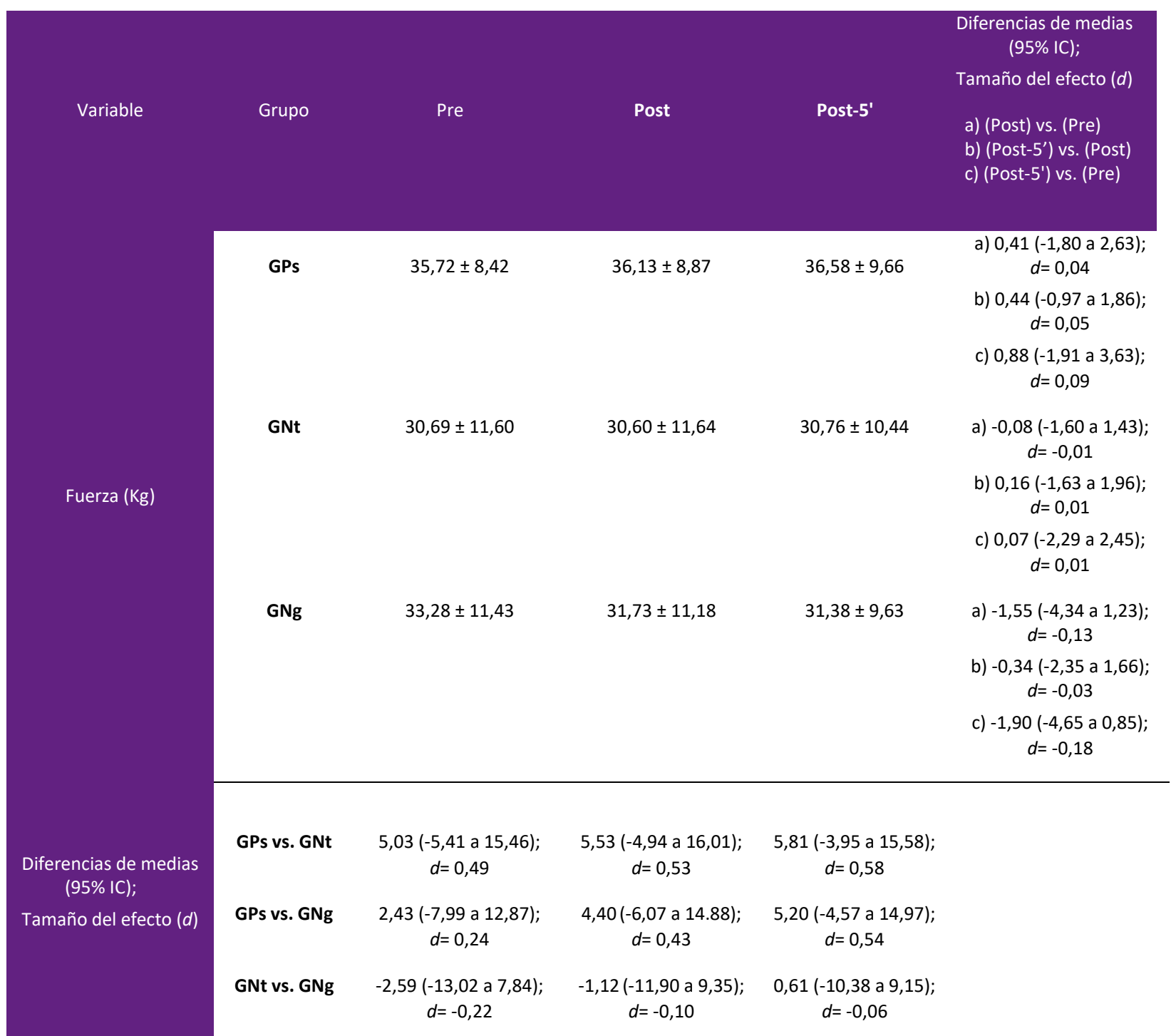

Abreviaturas: vs: versus; GPs: grupo de expectativas positivas; GNt: grupo de expectativas neutras; GNg: grupo de expectativas negativas; Kg: kilogramos; IC: intervalo de confianza.

$* \mathrm{p}<0,05$.

en la fuerza máxima que los sujetos eran capaces de producir, mientras que este aumento no se produjo en los sujetos que no tomaron la sustancia placebo. Por otro lado, Maganaris et al., (2000) observaron, en atletas profesionales, que tras la inducción de expectativas positivas con respecto a la ingesta de una sustancia anabolizante placebo consiguieron provocar un aumento de la fuerza máxima en el entrenamiento de levantamiento de peso.

Además, se reportó que este aumento se redujo tras eliminar la expectativa positiva además de informar a los sujetos de la composición real de la sustancia placebo. Los resultados obtenidos en el presente estudio coinciden parcialmente con los reportados anteriormente.

Los mecanismos neurofisiológicos subyacentes al posible aumento de la fuerza tras la inducción de expectativas positivas todavía permanecen inciertos y basados en hipótesis, sin embargo, se ha sugerido que, a nivel central, podría existir un proceso de liberación dopaminérgica, que, de forma subyacente, puede modular la actividad de las áreas relacionadas con la planificación y ejecución del movimiento voluntario, y en consecuencia, la actividad del tracto corticoespinal, pudiendo aumentar, a nivel periférico, el 
reclutamiento motor (Goetz et al., 2000; Parr \& Hyland, 2005). De la Fuente et al. (2001) trasladaron esta investigación básica a investigación clínica, obteniendo una hipótesis similar en pacientes con la enfermedad de Párkinson. En adición a esto, los autores del presente estudio también sugieren, que el efecto de la expectativa positiva podría estar mediado por la variable autoeficacia, a pesar de que esta no fue tomada en cuenta en la presente investigación.

Por otro lado, Peerdeman et al., (2015) observaron que la inducción de una expectativa positiva derivó en una disminución de la fatiga o la sensación de picazón en deportistas, poniendo de manifiesto la participación del sistema nervioso central en este tipo de percepciones físicas. Sugerimos por tanto que la inducción de expectativas puede tener una influencia en la fuerza ya que los resultados obtenidos en el presente estudio piloto, se muestra una tendencia positiva en este aspecto, aunque esta no sea estadísticamente significativa debido, probablemente, al tamaño muestral.

\section{Intensidad de dolor y variables somato-sensoriales}

En relación al papel de las expectativas sobre el dolor, Van Den Broeke et al. (2013) encontraron un aumento en los niveles de hiperalgesia mecánica tras la utilización de una corriente de alta frecuencia aplicada de forma tópica en sujetos sanos que habían sido expuestos a una expectativa negativa, mientras que los sujetos que no recibieron tal expectativa, presentaron menores niveles de la misma. Además, Cormier et al. (2013) han mostrado como la utilización de expectativas positivas es capaz de provocar una disminución en la intensidad del dolor percibida por sujetos sanos sometidos a un dolor evocado. Estos datos se corresponden con los obtenidos en el presente estudio.

Bialosky et al. $(2008,2009)$ hallaron cómo, tras la utilización de una técnica hipoalgesia como la manipulación de alta velocidad en la zona lumbar, ante la presencia de una expectativa negativa, se producía un aumento de la percepción dolorosa y, sin embargo, la misma técnica tras someter al participante a una expectativa positiva y neutra, acontecía un fenómeno de hipoalgesia local.

Con respecto a las variables somato-sensoriales, no se encontraron diferencias significativas ni en los UDP, ni en la sumación temporal en ninguno de los grupos de intervención. Se ha reportado en la literatura científica actual, que procesos de dolor persistente o de elevada intensidad pueden provocar la existencia de aberraciones somato-sensoriales (O’Neill et al., 2011; Hübscher et al., 2014). Los niveles de dolor post-intervención presentaban una intensidad menor a $30 \mathrm{~mm}$ en la escala de EVA. Se hipotetiza que si se hubiese utilizado una corriente TENS con efecto terapéutico en lugar de corriente placebo, quizá, al aumentar la intensidad, la irritación tópica hubiese sido mayor y hubiera podido dar lugar a cambios en dichas variables. Sin embargo, en relación a las expectativas y su influencia sobre estas son todavía inciertas y se requiere de mayor investigación que profundice en el tema.

\section{Limitaciones}

Este estudio presenta algunas limitaciones a tener en cuenta en la interpretación de los resultados. En primer lugar, el tamaño de la muestra es insuficiente para poder sacar conclusiones sólidas acerca de los resultados, y sería conveniente ampliarlo para futuros estudios. En segundo lugar, no fue posible conocer las expectativas previas de los participantes respecto al uso de una técnica de electroterapia, que es posible que pueda presentar una influencia en los resultados obtenidos. En tercer lugar, el estudio está realizado en sujetos sanos, por lo que existe la posibilidad que los resultados obtenidos difieran con la presencia de un proceso patológico que pueda mediar en las vías moduladoras descendentes o en los aspectos cognitivo o afectivos del paciente en los que se incluyen las expectativas. Por último, es posible que el corto tiempo de intervención pueda influir sobre las expectativas de los sujetos acerca del tratamiento, y hubiera sido interesante comprobar si otro tipo de metodología de intervención puede influir en la credibilidad de la intervención propuesta, afectando a la expectativa. 


\section{CONCLUSIÓN}

Los resultados del presente estudio piloto sugieren que las expectativas pueden tener una influencia en la fuerza de prensión palmar así como en la intensidad de dolor percibida post-intervención aunque estas diferencias no han mostrado ser estadísticamente significativas. Sin embargo, es necesario realizar un análisis con un tamaño muestral mayor para poder obtener conclusiones con mayor solidez. El papel de las expectativas sobre las variables somato-sensoriales es todavía incierto y se requiere de mayor investigación para poder evaluar dicha influencia.

\section{FRASES DESTACADAS}

$>$ El TENS aplicado no tuvo acción terapéutica para así observar analíticamente las expectativas.

$>$ Las expectativas positivas incrementaron ligeramente la fuerza de prensión manual.

$>$ Las expectativas negativas provocaron mayor dolor post-intervención.

$>$ A pesar de las tendencias observadas, no se hallaron cambios estadísticamente significativos.

\section{REFERENCIAS}

Bialosky JE, Bishop MD, Price DD, Robinson ME, George SZ. The mechanisms of manual therapy in the treatment of musculoskeletal pain: a comprehensive model. Man Ther. 2009;14(5):531-8.

DOI: http://dx.doi.org/10.1016/j.math.2008.09.001.

Bialosky JE, Bishop MD, Robinson ME, Barabas JA, George SZ. The influence of expectation on spinal manipulation induced hypoalgesia: an experimental study in normal subjects. BMC Musculoskelet Disord. 2008;9:19. DOI: http://dx.doi.org/10.1186/1471-2474-9-19.

Bijur PE, Silver W, Gallagher EJ. Reliability of the visual analog scale for measurement of acute pain. Acad Emerg Med. 2001;8(12):1153-7.

Campbell CM, Witmer K, Simango M, Carteret A, Loggia ML, Campbell JN, Haythornthwaite JA, Edwards RR. Catastrophizing delays the analgesic effect of distraction. Pain. 2010;149(2):202-7. DOI: http://dx.doi.org/10.1016/j.pain.2009.11.012.
Chesterton LS, Sim J, Wright CC, Foster NE. Interrater reliability of algometry in measuring pressure pain thresholds in healthy humans, using multiple raters. Clin $\mathrm{J}$ Pain. 2007;23(9):760-6. DOI: http://dx.doi.org/10.1097/AJP.0b013e318154b6ae.

Cohen, J. Statistical power analysis for the behavioral sciences, 2nd ed. Hillsdale, NJ; Lawrence Erlbaum Associates; 1988.

Cormier S, Piché M, Rainville P. Expectations modulate heterotopic noxious counter-stimulation analgesia. J Pain. 2013;14(2):114-25.

DOI: http://dx.doi.org/10.1016/j.jpain.2012.10.006.

De la Fuente-Fernández R, Ruth TJ, Sossi V, Schulzer M, Calne DB, Stoessl AJ. Expectation and dopamine release: mechanism of the placebo effect in parkinson's disease. Science. 2001;293(5532):1164-6. DOI: http://dx.doi.org/10.1126/science.1060937.

Deyo RA, Walsh NE, Martin DC, Schoenfeld LS, Ramamurthy S. A controlled trial of transcutaneous electrical nerve stimulation (TENS) and exercise for chronic low back pain. N Engl J Med. 1990;322(23):1627-34. DOI: http://dx.doi.org/10.1056/NEJM199006073222303.

Faasse K, Petrie KJ. The nocebo effect: patient expectations and medication side effects. Postgrad Med J. 2013;89(1055):540-6. DOI: http://dx.doi.org/10.1136/postgradmedj-2012-131730.

Fischer AA. Pressure algometry over normal muscles Standard values, validity and reproducibility of pressure threshold. Pain. 1987;30(1):115-26.

Fonseca-Pedrero E, Paino M, Sierra-Baigrie S, Lemos-Giráldez S, Muñiz J. Propiedades psicométricas del cuestionario de ansiedad estado-rasgo (STAI) en universitarios. Behav Psychol / Psicol Conduct. 2012;20(3):547-61.

García-Campayo J, Rodero B, Alda M, Sobradiel N, Montero J, Moreno S. Validación de la versión española de la escala de la catastrofización ante el dolor (Pain Catastrophizing Scale) en la fibromialgia. Medicina Clínica. 2008;(13):487-92. DOI: http://dx.doi.org/10.1157/13127277.

Goetz CG, Leurgans S, Raman R, Stebbins GT. Objective changes in motor function during placebo treatment in PD. Neurology. 2000;54(3):710-4.

Hübscher M, Moloney N, Rebbeck T, Traeger A, Refshauge KM. Contributions of mood, pain catastrophizing, and cold hyperalgesia in acute and chronic low back pain. Clin J Pain. 2014;30(10):886-93.

DOI: http://dx.doi.org/10.1097/AJP.0000000000000045.

Jensen JL, Marstrand PCD, Nielsen JB. Motor skill training and strength training are associated with different plastic changes in the central nervous system. J Appl Physiol. 2005;99(4):1558-68.

DOI: http://dx.doi.org/10.1152/japplphysiol.01408.2004.

Kalasountas V, Reed J, Fitzpatrick J. The effect of placeboinduced changes in expectancies on maximal force production in college students. J Appl Sport Psychol. 2007;19(1):116-24. DOI: http://dx.doi.org/10.1080/10413200601123736. 
Kalauokalani D, Cherkin DC, Sherman KJ, Koepsell TD, Deyo RA. Lessons from a trial of acupuncture and massage for low back pain: patient expectations and treatment effects. Spine (Phila Pa 1976). 2001;26(13):1418-24.

Maganaris CN, Collins D, Sharp M. Expectancy effects and strength training: do steroids make a difference? Sport Psychol. 2000;14(3):272-8. DOI: http://dx.doi.org/10.1123/tsp.14.3.272.

Noakes TD, St Clair Gibson A, Lambert E V. From catastrophe to complexity: a novel model of integrative central neural regulation of effort and fatigue during exercise in humans: summary and conclusions. $\mathrm{Br} \mathrm{J}$ Sports Med. 2005;39(2):120-4.

DOI: http://dx.doi.org/10.1136/bjsm.2003.010330.

O’Neill S, Kjær P, Graven-Nielsen T, Manniche C, ArendtNielsen L. Low pressure pain thresholds are associated with, but does not predispose for, low back pain. Eur Spine J. 2011;20(12):2120-5.

DOI: http://dx.doi.org/10.1007/s00586-011-1796-4.

Palmer ST, Martin DJ, Steedman WM, Ravey J. Alteration of interferential current and transcutaneous electrical nerve stimulation frequency: effects on nerve excitation. Arch Phys Med Rehabil. 1999;80(9):1065-71.

Papciak AS, Feuerstein M. Psychological factors affecting isokinetic trunk strength testing in patients with workrelated chronic low back pain. J Occup Rehabil. 1991;1(2):95-104.

DOI: http://dx.doi.org/10.1007/BF01073380.

Parr-Brownlie LC, Hyland BI. Bradykinesia induced by dopamine D2 receptor blockade is associated with reduced motor cortex activity in the rat. J Neurosci. 2005;25(24):5700-9. DOI: http://dx.doi.org/10.1523/JNEUROSCI.0523-05.2005.

Peerdeman KJ, van Laarhoven AI, Donders AR, Hopman MT, Peters ML, Evers AW. Inducing expectations for health: effects of verbal suggestion and imagery on pain, itch, and fatigue as indicators of physical sensitivity. PLoS One. 2015;10(10):e0139563.

DOI: http://dx.doi.org/10.1371/journal.pone.0139563.

Peolsson A, Hedlund R, Oberg B. Intra- and inter-tester reliability and reference values for hand strength. $\mathrm{J}$ Rehabil Med. 2001;33(1):36-41.

Ružić V, Ivanec D, Modić-Stanke K. Effect of expectation on pain assessment of lower- and higher-intensity stimuli. Scand J Pain. 2017;14:9-14. DOI: http://dx.doi.org/10.1016/j.sjpain.2016.09.013.
Saavedra-Hernández M, Castro-Sánchez AM, Cuesta-Vargas AI, Cleland JA, Fernández-de-las-Peñas C, Arroyo-Morales M. The contribution of previous episodes of pain, pain intensity, physical impairment, and pain-related fear to disability in patients with chronic mechanical neck pain. Am J Phys Med Rehabil. 2012;91(12):1070-6. DOI: http://dx.doi.org/10.1097/PHM.0b013e31827449a5.

Solé E, Castarlenas E, Sánchez-Rodríguez E, Galán S, de la Vega R, Jensen MP, Miró J. The reliability and validity of the spanish version of the fear of pain questionnaire. J Health Psychol. 2017.2 DOI: http://dx.doi.org/10.1177/1359105316686669.

Stark T, Walker B, Phillips JK, Fejer R, Beck R. Hand-held dynamometry correlation with the gold standard isokinetic dynamometry: a systematic review. PM\&R. 2011;3(5):4729. DOI: 10.1016/j.pmrj.2010.10.025.

Starkweather AR, Heineman A, Storey S, Rubia G, Lyon DE, Greenspan J, Dorsey SG. Methods to measure peripheral and central sensitization using quantitative sensory testing: A focus on individuals with low back pain. Appl Nurs Res. 2016;29:237-41. DOI: http://dx.doi.org/10.1016/j.apnr.2015.03.013.

Tsai M, Ogrodniczuk JS, Sochting I, Mirmiran J. Forecasting success: patients' expectations for improvement and their relations to baseline, process and outcome variables in group cognitive-behavioural therapy for depression. Clin Psychol Psychother. 2012:21(2):97-107. DOI: http://dx.doi.org/10.1002/cpp.1831.

Van Den Broeke E, Geene N, Van Rijn C, Oosterman J. Negative expectations facilitate mechanical hyperalgesia after highfrequency electrical stimulation of human skin. Eur J Pain. 2013:18(1):86-91. DOI: http://dx.doi.org/10.1002/j.15322149.2013.00342.x.

Verhagen AP, Karels CH, Schellingerhout JM, Willemsen SP, Koes BW, Bierma-Zeinstra SMA. Pain severity and catastrophising modify treatment success in neck pain patients in primary care. Man Ther. 2010;15(3):267-72. DOI: http://dx.doi.org/10.1016/j.math.2010.01.005.

Watson J, Ring D. Influence of psychological factors on grip strength. J Hand Surg Am. 2008;33(10):1791-5. DOI: http://dx.doi.org/10.1016/j.jhsa.2008.07.006.

Younger J, Gandhi V, Hubbard E, Mackey S. Development of the stanford expectations of treatment scale (SETS): a tool for measuring patient outcome expectancy in clinical trials. Clin Trials. 2012;9(6). 